\title{
Optimal Multi Zones Search Technique to Detect a Lost Target by Using $k$ Sensors
}

\author{
Mohamed Abd Allah El-Hadidy ${ }^{1,2, *}$, Hamdy M. Abou-Gabal ${ }^{1}$, Aya M. Gabr ${ }^{1}$ \\ ${ }^{1}$ Mathematics Department, Faculty of Science, Tanta University, Tanta 31527, Egypt \\ ${ }^{2}$ Mathematics and Statistics Department, College of Science, Taibah University, Yanbu, Saudi Arabia
}

\begin{abstract}
This paper presents the discrete search technique on multi zones to detect a lost target by using $k$ sensors. The search region is divided into $k$ zones. These zones contain an equal number of states (cells) not necessarily identical. Each zone has a one sensor to detect the target. The target moves over the cells according to a random process. We consider the searching effort as a random variable with a known probability distribution. The detection function with the discounted reward function in a certain state $j$ and time interval $i$ are given. The distribution of the optimal effort that minimizes the probability of undetection is obtained after solving a discrete stochastic optimization problem. An algorithm is constructed to obtain the optimal solution as in the numerical application.
\end{abstract}

Keywords Detection Model, Nonlinear Stochastic Programming Problem, Discrete Search Problem, Discounted Effort Reward Search

AMS 2010 subject classifications 90B22, 90B40, 90B50, 37A50, 60K25.

DOI: $10.19139 /$ soic-2310-5070-1136

\section{Introduction}

The searching for the lost targets which are either fixed or moving, is vital in numerous regular citizen and military applications. For example, the decision of penetrating profundities in the quest for an underground mineral, looking for the lost submarines and boats on or under the ocean, looking for a school of fish and searching for a plane in the sky [1-3]. The search theory has been studied in many variations. When the target is fixed or moves randomly on the real line, we get the so called linear search problem. This problem has an important application in our life. It might emerge in numerous true circumstances, for example, looking for a broken unit in a huge straight framework (electrical cables, phone lines and mining framework), searching for some data in a memory of PC tapes, etc., (see [4-12]. When the target is very important, we use the cooperative search technique. In an earlier work, this technique is used to maximize the probability of the target detection as in [13-21]. Many authors have been succeeded to apply the idea of the cooperative techniques on the plane and space, see [22-27,41,42]. The readers can find different techniques and models to detect the lost target in [28-36]. The main purpose of these different techniques is finding the target in minimum cost and with maximum probability. More recent search models to detect the lost targets can be found in $[44,45]$.

Sometimes the search region has various natural factors and we have a difficulty to apply the above techniques. Thus, the experts in this field divided the search region to a set of states (may be identical or not). In [38-40], the search region is divided into a finite set of square and identical cells. The lost target is randomly moving over these *Correspondence to: Mohamed Abd Allah El-Hadidy (Email: melhadidi@science.tanta.edu.eg). Mathematics Department, Faculty of
Science, Tanta University, Tanta 31527, Egypt.

ISSN 2310-5070 (online) ISSN 2311-004X (print)

Copyright (C) 2021 International Academic Press 
cells. They also studied a special case when the target is hidden in one cell of them. On the other hand, in [41] the search area is divided into hexagonal cells. They introduced an algorithm for the optimal search path.

In this paper, the search region is divided into $\mathrm{k}$ zones. These zones contain an equal number of cells not necessarily identical. Each zone contains $m$ cells. There are $k$ sensors, where each zone has one sensor $\hat{S}$. The lost target is assumed to be in one of several $\mathrm{km}$-states (cells). The sensor searches for this target during the time intervals $i=1,2, \cdots, X$. After the searching in any state, the sensor switch without any delay to another state in its zone. Also, we apply the discounted effort reward function which used in [38]. The main objective is to minimize the searching effort and maximize the probability of detection.

This paper is organized as follows: Section 2 presents the formulation of our model. Section 3 gives the minimum search effort after solving a difficult discrete stochastic optimization problem under the effect of the discounted effort reward function. We construct an algorithm to get this solution numerically as in Section 4. This numerical solution appears in Section 5 for a real life application. Section 6 discusses the results and the future work.

\section{Model Formulation}

To accelerate the target finding of the lost target in a region of varied terrain, we should divided it into a group of zones.

The space of search: The search region is divided into $k$ zones. These zones contain an equal number of states (cells) not necessarily identical, see Figure 1 . Each zone contains $m$ cells.

The means of search: To conduct the search technique within the cells, we need $k$ sensors where each zone has one sensor $\hat{S}$ to explore it.

The target: It is assumed to be in one of several $\mathrm{km}$-states (cells) not necessarily identical states. The sensor searches for this target during the time interval $i$. After the search in any state, the sensor switch without any delay to another state in its zone. Any sensor of them must distribute his effort among its states in such a manner as to minimize the probability of undetection. The target occupies one state during each of $X$ times intervals. The probabilities that the target exists in state $j$ in zone $\mathrm{s}$ at time interval $i$ is denoted by $p_{i j s}, j=1,2, \cdots, m$, $s=1,2, \ldots, k$. The Effort is given by $0 \leq L_{i}(W) \leq \sum_{\hat{S}=1}^{k} V_{i}^{(\hat{S})}$ which will be distributed among the states and its value bounded by a random variable $\sum_{\hat{S}=1}^{k} V_{i}^{(\hat{S})}$. The allocation of all search effort is $W_{i j s}^{(\hat{S})}$, where $i=1,2, \cdots, X$, $j=1,2, \cdots, m, \hat{S}=s=1,2, \cdots, k$, which gives the effort to be put in state $j$ in zone $s$ at time interval $i$ by the sensor $\hat{S}$.

We call $W=\left(W_{i j 1}^{(1)}, W_{i j 2}^{(2)}, \cdots, W_{i j k}^{(k)}\right)$ be a search plan. The conditional probability of detecting the target at time $i$ with $W_{i j s}^{(\hat{S})}$ amount of effort given that the target is located in state $j$ in zone $s$, is given by the detection function $\left(1-b\left(i, j, s, W_{i j s}^{(\hat{S})}\right)\right), i=1,2, \cdots, X, j=1,2, \cdots, m, s=1,2, \cdots, k$. We assume that the searches at distinct time intervals are independent and the motion of the target is independent of the sensors's actions.

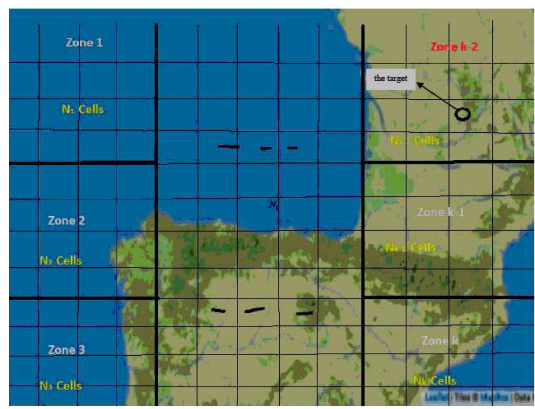

Figure 1. The Search region divided into $k$ zones with $k m$ cells. 


\section{Theorem 1}

The probability of undetection of the lost target over the whole time is given by:

$$
H(W)=\prod_{1=1}^{X}\left[\sum_{s=\hat{S}=1}^{k} \sum_{j=1}^{m} p_{i j s} b\left(i, j, s, W_{i j s}^{(\hat{S})}\right)\right] .
$$

Proof

$$
\begin{aligned}
H(W)= & {\left[p_{111} b\left(1,1,1, W_{111}^{(1)}\right)+p_{121} b\left(1,2,1, W_{121}^{(1)}\right)+\cdots+p_{1 m 1} b\left(1, m, 1, W_{1 m 1}^{(1)}\right)+p_{112} b\left(1,1,2, W_{112}^{(2)}\right)\right.} \\
& +p_{122} b\left(1,2,2, W_{122}^{(2)}\right)+\cdots+p_{1 m 2} b\left(1, m, 2, W_{1 m 2}^{(2)}\right)+\cdots+p_{11 k} b\left(1,1, k, W_{11 k}^{(k)}\right)+p_{12 k} b(1,2, k \\
& \left.\left.W_{12 k}^{(k)}\right)+\cdots+p_{1 m k} b\left(1, m, k, W_{1 m k}^{(k)}\right)\right] \times\left[p_{211} b\left(2,1,1, W_{211}^{(1)}\right)+p_{221} b\left(2,2,1, W_{221}^{(1)}\right)+\cdots\right. \\
& +p_{2 m 1} b\left(2, m, 1, W_{2 m 1}^{(1)}\right)+p_{212} b\left(2,1,2, W_{212}^{(2)}\right)+p_{222} b\left(2,2,2, W_{222}^{(2)}\right)+\cdots+p_{2 m 2} b\left(2, m, 2, W_{2 m 2}^{(2)}\right) \\
& \left.+\cdots+p_{21 k} b\left(2,1, k, W_{21 k}^{(k)}\right)+p_{22 k} b\left(2,2, k, W_{22 k}^{(k)}\right)+\cdots+p_{2 m k} b\left(2, m, k, W_{2 m k}^{(k)}\right)\right] \times \cdots \times \\
& {\left[p_{x 11} b\left(x, 1,1, W_{x 11}^{(1)}\right)+p_{x 21} b\left(x, 2,1, W_{x 21}^{(1)}\right)+\cdots+p_{x m 1} b\left(x, m, 1, W_{x m 1}^{(1)}\right)\right) p_{x 12} b\left(x, 1,2, W_{x 12}^{(2)}\right) } \\
& +p_{x 22} b\left(x, 2,2, W_{x 22}^{2)}\right)+\cdots+p_{x m 2} b\left(x, m, 2, W_{x m 2}^{(2)}\right)+\cdots+p_{x 1 k} b\left(x, 1, k, W_{x 1 k}^{(k)}\right)+p_{x 2 k} b(x, 2, k \\
& \left.\left.W_{x 2 k}^{(k)}\right)+\cdots+p_{x m k} b\left(x, m, k, W_{x m k}^{(k)}\right)\right]
\end{aligned}
$$

which can be written as $H(W)=\prod_{i=1}^{X}\left[\sum_{s=\hat{S}=1}^{k} \sum_{j=1}^{m} p_{i j s} b\left(i, j, s, W_{i j s}^{(\hat{S})}\right)\right]$.

In addition, we obtain the total effort from the following theorem.

\section{Theorem 2}

The total effort is given by:

$$
L(W)=\sum_{i=1}^{x} \sum_{s=\hat{S}=1}^{k} \sum_{j=1}^{m} W_{i j s}^{(\hat{S})}
$$

Proof

$$
\begin{aligned}
L(W)= & W_{111}^{(1)}+W_{121}^{(1)}+\cdots+W_{1 m 1}^{(1)}+W_{112}^{(2)}+W_{122}^{(2)}+\cdots+W_{1 m 2}^{(2)}+\cdots+W_{11 k}^{(k)}+W_{12 k}^{(k)}+\cdots+W_{1 m k}^{(k)} \\
& +W_{211}^{(1)}+W_{221}^{(1)}+\cdots+W_{2 m 1}^{(1)}+W_{212}^{(2)}+W_{222}^{(2)}+\cdots+W_{2 m 2}^{(2)}+\cdots+W_{21 k}^{(k)}+W_{22 k}^{(k)}+\cdots+W_{2 m k}^{(k)} \\
& +\cdots+W_{x 11}^{(1)}+W_{x 21}^{(1)}+\cdots+W_{x m 1}^{(1)}+W_{x 12}^{(2)}+W_{x 22}^{(2)}+\cdots+W_{x m 2}^{(2)}+\cdots+W_{x 1 k}^{(k)}+W_{x 2 k}^{(k)} \\
& +\cdots+W_{x m k}^{(k)} .
\end{aligned}
$$

Hence, $L(W)=\sum_{i=1}^{x} \sum_{s=\hat{S}=1}^{k} \sum_{j=1}^{m} W_{i j s}^{(\hat{S})}$.

The detection function: We consider the setection function is exponential, that is $1-b\left(i, j, s, W_{i j s}^{(\hat{S})}\right)=\frac{1}{k}(1-$ $\left.e^{\frac{-W_{i j s}^{(\hat{S})}}{T_{j s}}}\right), i=1,2, \cdots, X, j=1,2, \cdots, m, s=\hat{S}=1,2, \cdots, k$, where $T_{j s}$ is a factor due to the search in cell $j$ in zone $s$, and the dimensions of it, then the probability of undetection of the target over the whole time is given by,

$$
H(W)=\prod_{i=1}^{X}\left[\sum_{s=\hat{S}=1}^{k} \sum_{j=1}^{m} p_{i j s}\left(1-\frac{1}{k}\left(1-e^{\frac{-W_{i j s}^{(\hat{S})}}{T_{j s}}}\right)\right)\right],
$$


and the unrestricted effort is given by:

$$
L(W)=\sum_{i=1}^{X} \sum_{s=\hat{S}=1}^{k} \sum_{j=1}^{m} W_{i j s}^{(\hat{S})} \leq \sum_{i=1}^{X} \sum_{s=\hat{S}=1}^{k} V_{i}^{(\hat{S})}=V, \quad L_{i}(W) \leq \sum_{s=\hat{S}=1}^{k} V_{i}^{(\hat{S})}
$$

Let $\sum_{s=\hat{S}=1} V_{i}^{(\hat{S})}$ be a random variable with normal distribution and it has a probability density function $f\left(\sum_{s=\hat{S}=1}^{k} V_{i}^{(\hat{S})}\right)$ and the distribution function $F\left(\sum_{s=\hat{S}=1}^{k} V_{i}^{(\hat{S})}\right), i=1,2,3, \cdots, X$.

Our aim is to find $W=\left(W_{i j 1}^{(1)}, W_{i j 2}^{(2)}, \cdots, W_{i j k}^{(k)}\right)$ which minimize $H(W)$ subject to the constraints: $\left.L_{i}(W) \leq \sum_{s=\hat{S}=1}^{k} V_{i}^{(\hat{S})}, 0 \leq W_{i j s}^{(k)}\right)$ and $\sum_{s=1}^{k} \sum_{j=1}^{m} p_{i j s}=1$, where $W$ is a function of $\left(\sum_{s=\hat{S}=1}^{k} V_{i}^{(\hat{S})}\right)$. Since, the detection function is exponential then the problem will become a convex nonlinear programming problem (NLP) as follows:

NLP :

$$
\min _{W_{i j s}^{(\hat{S})}} H(W)=\prod_{i=1}^{X}\left[\sum_{s=\hat{S}=1}^{k} \sum_{j=1}^{m} p_{i j s}\left(1-\frac{1}{k}\left(1-e^{\frac{-W_{i j s}^{(\hat{S})}}{T j s}}\right)\right)\right]
$$

subject to

$$
\begin{aligned}
& W\left(\sum_{s=\hat{S}=1}^{k} V_{i}^{(\hat{S})}\right)=\left\{W \in R^{X k m} / L_{i}(W) \leq W\left(\sum_{s=\hat{S}=1}^{k} V_{i}^{(\hat{S})}\right),\right. \\
& \left.L(W)=\sum_{i=1}^{X} \sum_{s=\hat{S}=1}^{k} \sum_{j=1}^{m} W_{i j s}^{(\hat{S})} \leq \sum_{i=1}^{X} \sum_{s=\hat{S}=1}^{k} V_{i}^{(\hat{S})}\right\}, \\
& 0 \leq W_{i j s}^{(\hat{S})} \forall i=1,2, \cdots, x, j=1,2, \cdots, m, s=\hat{S}=1,2, \cdots, k \text { and } \sum_{s=\hat{S}=1}^{k} \sum_{j=1}^{m} p_{i j s}=1
\end{aligned}
$$

where $R^{X k m}$ is the feasible set of constrained decisions. The unique solution is guaranteed by the convexisty of $H(W)$ and $W\left(\sum_{s=\hat{S}=1}^{k} V_{i}^{(\hat{S})}\right)$.

\section{Definition 1}

$\bar{W} \in W\left(\sum_{s=\hat{S}=1}^{k} V_{i}^{(\hat{S})}\right)$ is said to be an optimal solution for problem (5) if there does not exists $W \in$ $W\left(\sum_{s=\hat{S}=1}^{k} V_{i}^{(\hat{S})}\right)$ such that $H(W) \leq H(\bar{W})$ with at least one strict inequality holds, with $\left(L_{i}(W) \leq\right.$ $\left.\sum_{s=\hat{S}=1}^{k} V_{i}^{(\hat{S})}\right) \leq \beta, \beta \in[0,1]$. 
The corresponding nonlinear stochastic programming problem (NLP) is:

NLP :

$\min _{W_{i j s}^{(\hat{S})}} H(W)=\prod_{i=1}^{X}\left[\sum_{s=\hat{S}=1}^{k} \sum_{j=1}^{m} p_{i j s}\left(1-\frac{1}{k}\left(1-e^{\frac{-w_{i j s}^{(\hat{S})}}{T j s}}\right)\right)\right]$,

subject to

$$
\begin{aligned}
& P\left(L_{i}(W) \leq \sum_{s=\hat{S}=1}^{k} V_{i}^{(\hat{S})}\right) \leq \beta, \quad \beta \in[0,1], \\
& 0 \leq W_{i j s}^{(\hat{S})} \forall i=1,2, \cdots, X, \quad j=1,2, \cdots, m, \quad s=\hat{S}=1,2, \cdots, k \quad \text { and } \quad \sum_{s=1}^{k} \sum_{j=1}^{m} p_{i j s}=1 .
\end{aligned}
$$

The constraints $\widetilde{P}\left(L_{i}(W) \leq \sum_{s=\hat{S}=1}^{k} V_{i}^{(\hat{S})} \geq 1-\beta\right.$ has to satisfied with unprobability of at least $(1-\beta)$ and can be restated as:

$$
\widetilde{P}\left(\frac{L_{i}(W)-E\left(\sum_{s=\hat{S}=1}^{k} V_{i}^{(\hat{S})}\right)}{\sqrt{\operatorname{var}\left(\sum_{s=\hat{S}=1}^{k} V_{i}^{(\hat{S})}\right)}} \leq \frac{\left(\sum_{s=\hat{S}=1}^{k} V_{i}^{(\hat{S})}\right)-E\left(\sum_{s=\hat{S}=1}^{k} V_{i}^{(\hat{S})}\right)}{\sqrt{\operatorname{var}\left(\sum_{s=\hat{S}=1}^{k} V_{i}^{(\hat{S})}\right)}}\right) \geq 1-\beta,
$$

and for the complement probability we have:

$$
\widetilde{P}\left(\frac{L_{i}(W)-E\left(\sum_{s=\hat{S}=1}^{k} V_{i}^{(\hat{S})}\right)}{\sqrt{\operatorname{var}\left(\sum_{s=\hat{S}=1}^{k} V_{i}^{(\hat{S})}\right)}} \geq \frac{\left(\sum_{s=\hat{S}=1}^{k} V_{i}^{(\hat{S})}\right)-E\left(\sum_{s=\hat{S}=1}^{k} V_{i}^{(\hat{S})}\right)}{\sqrt{\operatorname{var}\left(\sum_{s=\hat{S}=1}^{k} V_{i}^{(\hat{S})}\right)}}\right) \leq \beta,
$$

where $\frac{\left(\sum_{s=\hat{S}=1}^{k} V_{i}^{(\hat{S})}\right)-E\left(\sum_{s=\hat{S}=1}^{k} V_{i}^{(\hat{S})}\right)}{\sqrt{\operatorname{var}\left(\sum_{s=\hat{S}=1}^{k} V_{i}^{(\hat{S})}\right)}}$ is a standard normal random variable.

If $K_{p}$ represent the value of the standard normal random variable at which $\varphi\left(K_{p}\right)=\beta$, then this constraint can be expressed as:

$$
\varphi\left(\frac{L_{i}(W)-E\left(\sum_{s=\hat{S}=1}^{k} V_{i}^{(\hat{S})}\right)}{\sqrt{\operatorname{var}\left(\sum_{s=\hat{S}=1}^{k} V_{i}^{(\hat{S})}\right)}}\right) \leq \varphi\left(K_{p}\right)
$$

this inequality will be satisfied only if:

$$
L_{i}(W)-E\left(\sum_{s=\hat{S}=1}^{k} V_{i}^{(\hat{S})}\right) \leq K_{p} \sqrt{\operatorname{var}\left(\sum_{s=\hat{S}=1}^{k} V_{i}^{(\hat{S})}\right)} .
$$


Suppose that the probability of undetection (3) is combined with the discounted effort function $0<\delta_{j s}^{i}<1$ which used in El-Hadidy [38,39] to develop the final discounted effort reward function:

$$
H(W)=\prod_{i=1}^{X}\left[\sum_{s=\hat{S}=1}^{k} \sum_{j=1}^{m}\left(1-\delta_{j s}^{i}\right) p_{i j s}\left(1-\frac{1}{k}\left(1-e^{\frac{-W_{i j s}^{(\hat{S})}}{T_{j s}}}\right)\right)\right],
$$

thus, the NLSP problem (6) is equivalent to the following deterministic nonlinear stochastic programming problem (DNLSP),

DNLSP :

$$
\min _{W_{i j s}^{(\hat{S})}} H(W)=\prod_{i=1}^{X}\left[\sum_{s=\hat{S}=1}^{k} \sum_{j=1}^{m}\left(1-\delta_{j s}^{i}\right) p_{i j s}\left(1-\frac{1}{k}\left(1-e^{\frac{-W_{i j s}^{(\hat{S})}}{T j s}}\right)\right)\right],
$$

subject to

$$
\begin{gathered}
L_{i}(W)-E\left(\sum_{s=\hat{S}=1}^{k} V_{i}^{(\hat{S})}\right) \leq K_{p} \sqrt{\operatorname{var}\left(\sum_{s=\hat{S}=1}^{k} V_{i}^{(\hat{S})}\right)} \\
0 \leq W_{i j s}^{(\hat{S})}, \quad 0<\delta_{j s}^{i}<1 \forall i=1,2, \cdots, X, j=1,2, \cdots, m, s=1,2, \cdots, k \text { and } \sum_{s=1}^{k} \sum_{j=1}^{m} p_{i j s}=1 .
\end{gathered}
$$

which equivalent to:

$$
\min _{W_{i j s}^{(\hat{S})}, \delta_{j s}^{i}} H(W)=\prod_{i=1}^{X}\left[\sum_{s=\hat{S}=1}^{k} \sum_{j=1}^{m}\left(1-\delta_{j s}^{i}\right) p_{i j s}\left(1-\frac{1}{k}\left(1-e^{\frac{-W_{i j s}^{(\hat{S})}}{T j s}}\right)\right)\right],
$$

subject to

$$
\begin{aligned}
& W\left(\sum_{s=\hat{S}=1}^{k} V_{i}^{(\hat{S})}\right)=\left\{W \in R^{X k m} / g(w)=\sum_{s=\hat{S}=1}^{k} \sum_{j=1}^{m} W_{i j s}^{(\hat{S})}-E\left(\sum_{s=\hat{S}=1}^{k} V_{i}^{(\hat{S})}\right)\right. \\
&\left.-K_{p} \sqrt{\operatorname{var}\left(\sum_{s=\hat{S}=1}^{k} V_{i}^{(\hat{S})}\right)} \leq 0\right\}, \\
&-W_{i j s}^{(\hat{S})} \leq 0,-\delta_{j s}^{i}<0, p_{i j s}-1 \leq 0 \forall i=1,2, \cdots, X, j=1,2, \cdots, m, s=1,2, \cdots, k .
\end{aligned}
$$

\section{The Minimum Search Effort}

The main purpose here is to minimize the searching effort under the above constraints. Since, the detection function is convex then one can apply the necessary Kuhn-Tucker conditions to solve the problem (9). Consequently, we get:

$$
\begin{gathered}
-\frac{\left(1-\delta_{j s}^{\sigma}\right) p_{\sigma j s}}{k T_{j s}} e^{-\left(W_{\sigma j s}^{(\hat{S})} / T_{j s}\right)} \prod_{\substack{i=1 \\
i \neq \sigma}}^{X} \sum_{s=\hat{S}=1}^{k} \sum_{j=1}^{m}\left(1-\delta_{j s}^{i}\right) p_{i j s}\left(1-\frac{1}{k}\left(1-e^{-\frac{W_{i j s}^{(\hat{S})}}{T_{j s}}}\right)\right)+U_{1 \sigma}-U_{2 \sigma}=0 \\
-\sigma \delta_{j s}^{\sigma-1} \prod_{\substack{i=1 \\
i \neq \sigma}}^{X} \sum_{s=\hat{S}=1}^{k} \sum_{j=1}^{m}\left(1-\delta_{j s}^{i}\right) p_{i j s}\left(1-\frac{1}{k}\left(1-e^{-\frac{W_{i j s}^{(\hat{S})}}{T_{j s}}}\right)\right)-U_{3 \sigma}=0,
\end{gathered}
$$




$$
\begin{gathered}
U_{1 \sigma}\left\{\sum_{s=\hat{S}=1}^{k} \sum_{j=1}^{m} W_{\sigma j s}^{(\hat{S})}-E\left(\sum_{s=\hat{S}=1}^{k} v_{\sigma}^{(\hat{S})}\right)-K_{p} \sqrt{\operatorname{Var}\left(\sum_{s=\hat{S}=1}^{k} v_{\sigma}^{(\hat{S})}\right)}\right\}=0, \\
U_{2 \sigma}\left\{-W_{\sigma j s}^{(\hat{S})}\right\}=0, \\
U_{3 \sigma}\left\{-\delta_{j s}^{\sigma}\right\}=0,
\end{gathered}
$$

where $U_{\xi \sigma, \xi}=1,2,3$ is the Lagrange multiplies. Since $W_{\sigma j s}^{(\hat{S})}>0$ and $\delta_{j s}^{\sigma}>0$ then from (13) and (14) we have $U_{2 \sigma}=U_{3 \sigma}=0$. Also, $\sum_{j=1}^{m} \sum_{s=\hat{S}=1}^{k} W_{\sigma j s}^{(\hat{S})}-E\left(\sum_{s=\hat{S}=1}^{k} v_{\sigma}^{(\hat{S})}\right)-K_{p} \sqrt{\operatorname{Var}\left(\sum_{s=\hat{S}=1}^{k} v_{\sigma}^{(\hat{S})}\right)}<0$, then from (12), one can get $U_{1 \sigma} \neq 0$. Thus, the case $U_{1 \sigma}>0, U_{2 \sigma}=U_{3 \sigma}=0$ is the only case which consider to get the minimum searching effort $\left(W_{\sigma j s}^{(\hat{S})}\right)^{*}$ and the optimal value $\left(\delta_{j s}^{\sigma}\right)^{*}$. Thus, from (12) we have:

$$
U_{1 \sigma}=\frac{\left(1-\delta_{j s}^{\sigma}\right) p_{\sigma j s}}{k T_{j s}} e^{-\frac{W_{\sigma j s}^{(\hat{S})}}{T_{j s}}} \prod_{\substack{i=1 \\ i \neq \sigma}}^{X} \sum_{s=\hat{S}=1}^{k} \sum_{j=1}^{m}\left(1-\delta_{j s}^{i}\right) p_{i j s}\left(1-\frac{1}{k}\left(1-e^{-\frac{W_{i j s}^{(\hat{S})}}{T_{j s}}}\right)\right) .
$$

From (15) in (12), we get:

$$
\begin{aligned}
& \left(\frac{\left(1-\delta_{j s}^{\sigma}\right) p_{\sigma j s}}{k T_{j s}} e^{-\frac{W_{\sigma j s}^{(\hat{S})}}{T_{j s}}} \prod_{\substack{i=1 \\
i \neq \sigma}}^{X} \sum_{s=\hat{S}=1}^{k} \sum_{j=1}^{m}\left(1-\delta_{j s}^{i}\right) p_{i j s}\left(1-\frac{1}{k}\left(1-e^{-\frac{W_{i j s}^{(\hat{S})}}{T_{j s}}}\right)\right)\right) \times\left\{\sum_{s=\hat{S}=1}^{k} \sum_{j=1}^{m} W_{\sigma j s}^{(\hat{S})}\right. \\
& \left.-E\left(\sum_{s=\hat{S}=1}^{k} v_{\sigma}^{(\hat{S})}\right)-K_{p} \sqrt{\operatorname{Var}\left(\sum_{s=\hat{S}=1}^{k} v_{\sigma}^{(\hat{S})}\right)}\right\}=0 .
\end{aligned}
$$

Thus,

$$
\frac{\left(1-\delta_{j s}^{\sigma}\right) p_{\sigma j s}}{k T_{j s}} e^{-\frac{W_{\sigma j s}^{(\hat{S})}}{T_{j s}}}=0
$$

or

$$
\prod_{\substack{i=1 \\ i \neq \sigma}}^{X} \sum_{s=\hat{S}=1}^{k} \sum_{j=1}^{m}\left(1-\delta_{j s}^{i}\right) p_{i j s}\left(1-\frac{1}{k}\left(1-e^{-\frac{W_{i j s}^{(\hat{S})}}{T_{j s}}}\right)\right)=0
$$

or

$$
\left\{\sum_{s=\hat{S}=1}^{k} \sum_{j=1}^{m} W_{\sigma j s}^{(\hat{S})}-E\left(\sum_{s=\hat{S}=1}^{k} v_{\sigma}^{(\hat{S})}\right)-K_{p} \sqrt{\operatorname{Var}\left(\sum_{s=\hat{S}=1}^{k} v_{\sigma}^{(\hat{S})}\right)}\right\}=0 .
$$

Put $r_{i}=E\left(\sum_{s=\hat{S}=1}^{k} V_{i}^{(\hat{S})}\right)+K_{p} \sqrt{\operatorname{Var}\left(\sum_{s=\hat{S}=1}^{k} V_{i}^{(\hat{S})}\right)}$, then from Equation (12) we obtain

$$
\sum_{s=\hat{S}=1}^{k} \sum_{j=1}^{m} W_{\sigma j s}^{(\hat{S})}-r_{i}=0
$$


at least one of these boundaries satisfies that,

$$
W_{i j s}^{(\hat{S})}-r_{i}=0 .
$$

Also, from (12), we conclude that at least one of these boundaries satisfies such that

$$
\left(1-\delta_{j s}^{i}\right) p_{i j s}\left(1-\frac{1}{k}\left(1-e^{-\frac{W_{i j s}^{(\hat{S})}}{T_{j s}}}\right)\right)=0
$$

Hence,

$$
\begin{aligned}
& \left(\frac{\left(1-\delta_{j s}^{\sigma}\right) p_{\sigma j s}}{k T_{j s}} e^{-\frac{W_{\sigma j s}^{(\hat{S})}}{T_{j s}}} \prod_{\substack{i=1 \\
i \neq \sigma}}^{X} \sum_{s=\hat{S}=1}^{k} \sum_{j=1}^{m}\left(1-\delta_{j s}^{i}\right) p_{i j s}\left(1-\frac{1}{k}\left(1-e^{-\frac{W_{i j s}^{(\hat{S})}}{T_{j s}}}\right)\right)\right) \cdot\left\{\sum_{s=\hat{S}=1}^{k} \sum_{j=1}^{m} W_{\sigma j s}^{(\hat{S})}\right. \\
& \left.-E\left(\sum_{s=\hat{S}=1}^{k} v_{\sigma}^{(\hat{S})}\right)-K_{p} \sqrt{\operatorname{Var}\left(\sum_{s=\hat{S}=1}^{k} v_{\sigma}^{(\hat{S})}\right)}\right\} \\
& =-\sigma \delta_{j s}^{\sigma-1} \prod_{\substack{i=1 \\
i \neq \sigma}}^{X} \sum_{s=\hat{S}=1}^{k} \sum_{j=1}^{m}\left(1-\delta_{j s}^{i}\right) p_{i j s}\left(1-\frac{1}{k}\left(1-e^{-\frac{W_{i j s}^{(\hat{S})}}{T_{j s}}}\right)\right)=0
\end{aligned}
$$

This leads to,

$$
\left(\frac{\left(1-\delta_{j s}^{\sigma}\right) p_{\sigma j s}}{k T_{j s}} e^{-\frac{W_{\sigma j s}^{(\hat{S})}}{T_{j s}}} \cdot\left\{\sum_{s=\hat{S}=1}^{k} \sum_{j=1}^{m} W_{\sigma j s}^{(\hat{S})}-E\left(\sum_{s=\hat{S}=1}^{k} v_{\sigma}^{(\hat{S})}\right)-K_{p} \sqrt{\operatorname{Var}\left(\sum_{s=\hat{S}=1}^{k} v_{\sigma}^{(\hat{S})}\right)}\right\}+\sigma \delta_{j s}^{\sigma-1}=0 .\right.
$$

By solving (22) numerically, one can get the minimum value $\left(W_{\sigma j s}^{(\hat{S})}\right)^{*}$ and the optimal value $\left(\delta_{j s}^{\sigma}\right)^{*}$.

\section{Algorithm}

We construct the following algorithm to calculate the minimum search effort. The steps of the algoritm can be summarized as follows:

Step 1. Input the values of the following:

$k \quad$ is the number of zones,

$m$ is the number of cells in each zone,

$X$ is the total time intervals,

$p \quad$ the probability that the target exists in state $j$ in zone $s$ at time interval $i$,

$T_{j s} \quad$ is a factor due to the search in cell $j$ in zone $s$ and the dimensions of it, where $j=1,2, \cdots, m$, $s=\hat{S}=1,2, \cdots, k$,

$K_{p} \quad$ is the value of the standard normal random variable,

$E\left(\sum_{s=\hat{S}=1}^{k} V_{i}^{(\hat{S})}\right) \quad$ is the expected value of $\sum_{s=\hat{S}=1}^{k} V_{i}^{(\hat{S})}$,

$\operatorname{var}\left(\sum_{s=\hat{S}=1}^{k} V_{i}^{(\hat{S})}\right)$ is the variance of $\sum_{s=\hat{S}=1}^{k} V_{i}^{(\hat{S})}$.

Step 2. Compute the values of $\delta_{j s}^{i}$ from Equation (17), 
Step 3. Compute the values of $r_{i}$ from the relation $r_{i}=E\left(\sum_{s=\hat{S}=1}^{k} V_{i}^{(\hat{S})}\right)+K_{p} \sqrt{\operatorname{var}\left(\sum_{s=\hat{S}=1}^{k} V_{i}^{(\hat{S})}\right)}$, $\forall i=1,2, \cdots, X, s=\hat{S}=1,2, \cdots, k$, otherwise, go to step 8,

Step 4. Compute the values of $W_{j s}^{(\hat{S})}$ from Equation (20) otherwise, go to step 8,

Step 5. Substitute with the value of $\delta_{j s}^{i}, W_{i j s}^{(\hat{S})}, p_{i j s}$ and $r_{i}$ in Equation (7) to compute the value of $H(W)$,

Step 6. Replace $j$ by $j+1$, if $j \leq m$, then return to step 2 , and replace $i$ by $i+1$ and test the condition $i \leq X$, if yes then go step 2, otherwise, go to step 7 ,

Step 7. Give the total value of $H(W)$ and then stop.

Step 8. End.

\section{Application}

In this section, we apply the above algorithm to formulate a probabilistic search model to detect the randomly moving target in one of several different zones. Assume that, we have 5 zones that are divided into a total number of $N=30,40,50$ and 60 cells. For example, in the case of 30 cells (total number of cells), each zone will have 6 cells. In addition, each zone contains one sensor and there is no interference between them. The target moves randomly between these zones. At a random time interval, we consider the probability $p_{\sigma j s}$ that the target exists in state $j$ in zone $s$ at time interval $\sigma$ is randomly generated by using Maple 13. Also the parameters $T_{j s}$ and $r_{i}$ are randomly generated, see Table 1 . After applying the above algorithm, the minimum value of the search effort $\left(W_{\sigma j s}^{(\hat{S})}\right)^{*}$ and the optimal value $\left(\delta_{j s}^{\sigma}\right)^{*}$ are appearing in Table (1) in each case.

If we substitute with these random generated values in (22), then we have an infinite number of solutions which give the optimal values $\left(\delta_{j s}^{\sigma}\right)^{*}$ and $\left(W_{\sigma j s}^{(\hat{S})}\right)^{*}$. This appears in Figure (2). It presents the plotting $3 D$ of the relationship between $\delta_{j s}^{\sigma}$ and $W_{\sigma j s}^{(\hat{S})}$. The solution of (22) is an interested curve produced from the two intersecting planes and it is satisfied (22) (i.e., an infinite number of solution). We use Maple 13 to deduce the equation of the intersected curve as in Figure (3). This figure gives the value of $\left(\delta_{j s}^{\sigma}\right)^{*}$ which satisfies (22) and hence we deduce the minimum value $W_{\sigma j s}^{(\hat{S})}$ for each $N$.

Table 1 . The randomly generated values of $\sigma, r_{\sigma}, T_{j s}$ and $p_{\sigma j s}$ which give the optimal values of $\left(\delta_{j s}^{\sigma}\right)^{*}$ and $\left(W_{\sigma j s}^{(\hat{S})}\right)^{*}$ in $k=5,10$ zones.

\begin{tabular}{|c|c|c|c|c|c|c|c|c|}
\hline \multirow{2}{*}{$N$} & $\sigma$ & \multirow{2}{*}{$r_{\sigma}$} & \multirow{2}{*}{$T_{j s}$} & $p_{\sigma j s}$ & \multicolumn{2}{|c|}{5 zones } & \multicolumn{2}{|c|}{10 zones } \\
\cline { 6 - 9 } & & & & $\left(\delta_{j s}^{\sigma}\right)^{*} \cong$ & $W_{\sigma j s}^{(\hat{S})} \cong$ & $\left(\delta_{j s}^{\sigma}\right)^{*} \cong$ & $W_{\sigma j s}^{(\hat{S})} \cong$ \\
\hline 30 & 19 & 5.279190642 & 747.1503308 & 0.0334672756 & 0.484 & 0.04071571836 & 4.66 & 0.0381033075 \\
\hline 40 & 27 & 8.668184741 & 497.2015204 & 0.9669052975 & 0.705 & 0.03057684590 & 0.688 & 0.0130959880 \\
\hline 50 & 4 & 4.301371693 & 866.8184741 & 0.8675579182 & 0.0573 & 0.1353252176 & 0.0458 & 0.1153107240 \\
\hline 60 & 50 & 7.596612973 & 867.5579182 & 0.4301371693 & 0.796 & 0.01119397377 & 0.781 & 0.0411131617 \\
\hline
\end{tabular}

From Table (1), one can conclude that the region which divided into 5 zones has the maximum discounted effort reward parameters rather than 10 zones for the same number of cells. In general, we notice that, when the region is divide to 10 zones then the searching effort equals approximately the searching effort in the 5 zones case. In the 5 zones case, we used a small number of sensors. All of these results lead us to the result; that is the case of 5 zones will give the minimum value of $H(W)$ with maximum value $\left(\delta_{j s}^{\sigma}\right)^{*}$. 


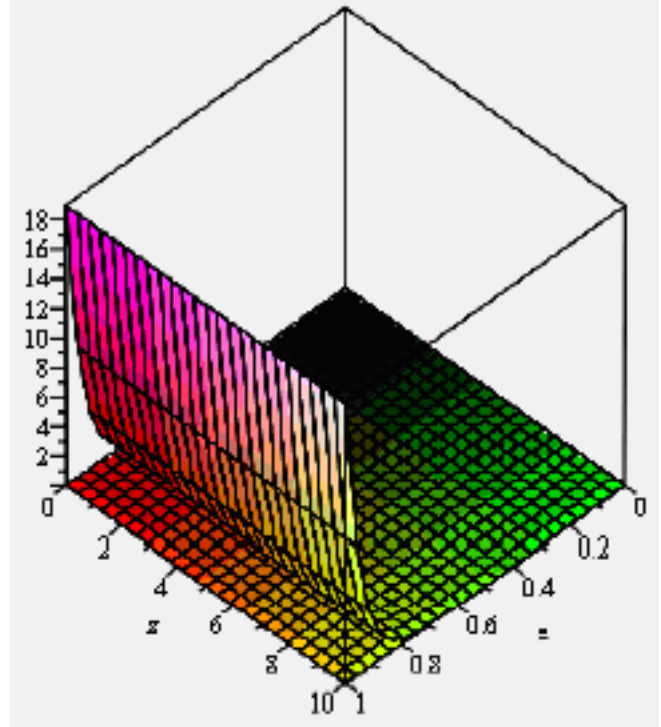

(a) $\quad N=30$

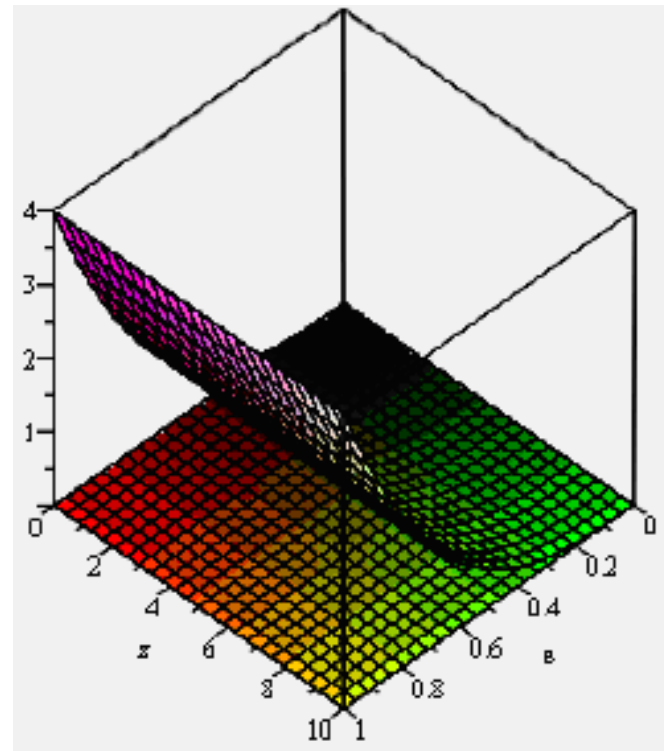

(c) $\quad N=50$

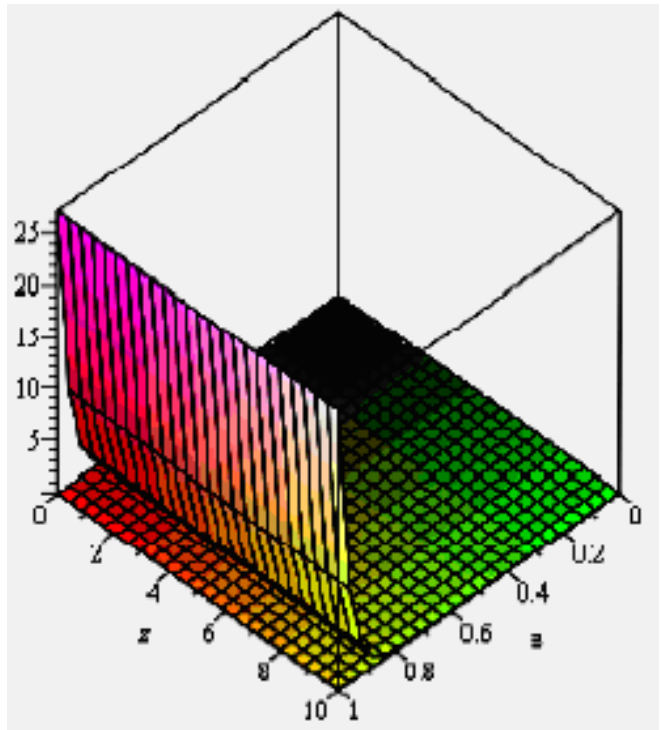

(b) $N=40$

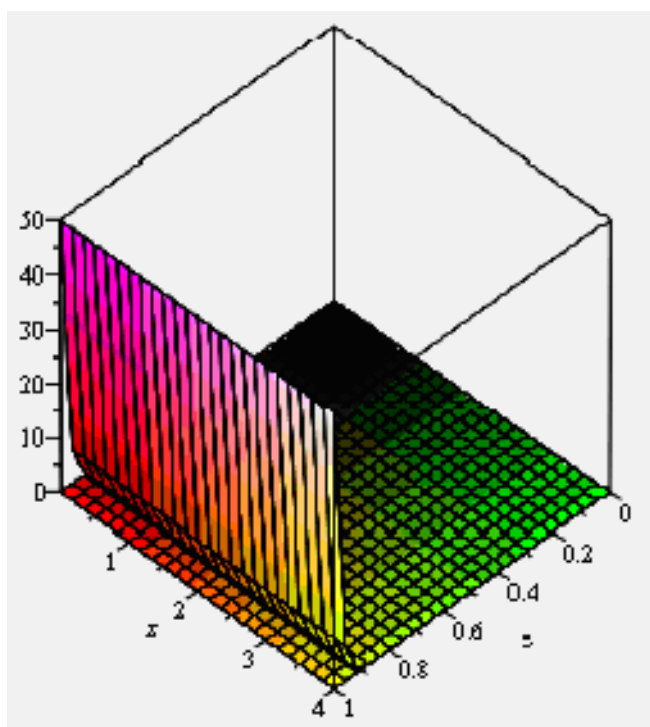

(d) $\quad N=60$

Figure 2. Plotting $3 D$ which presents the solution of (22) in 5 zones and different values of $N$. 


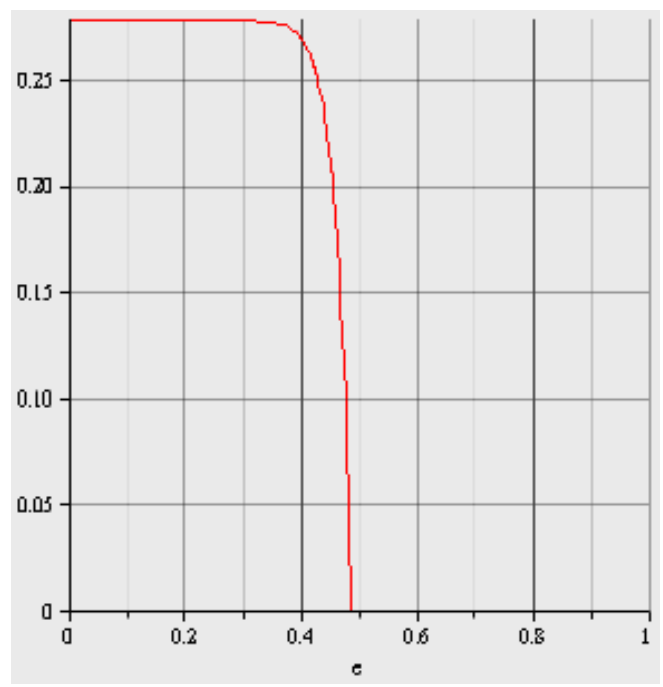

(a) $\quad N=30$

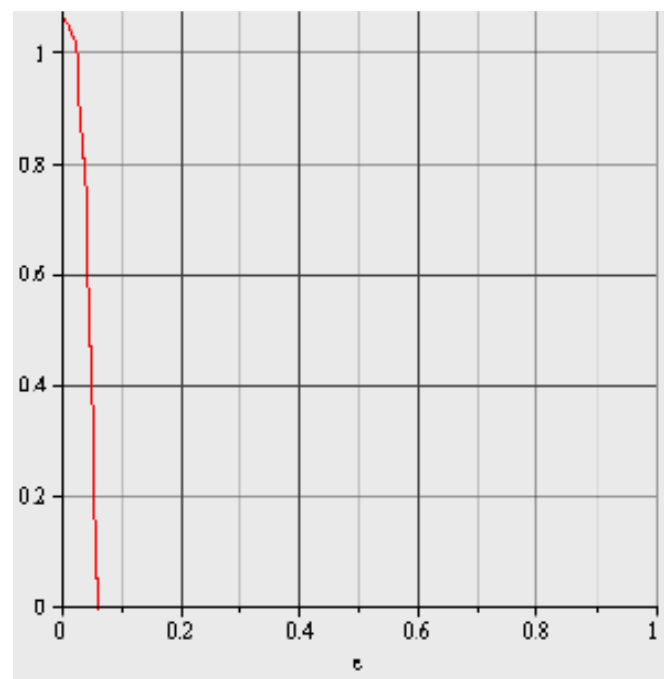

(c) $\quad N=50$

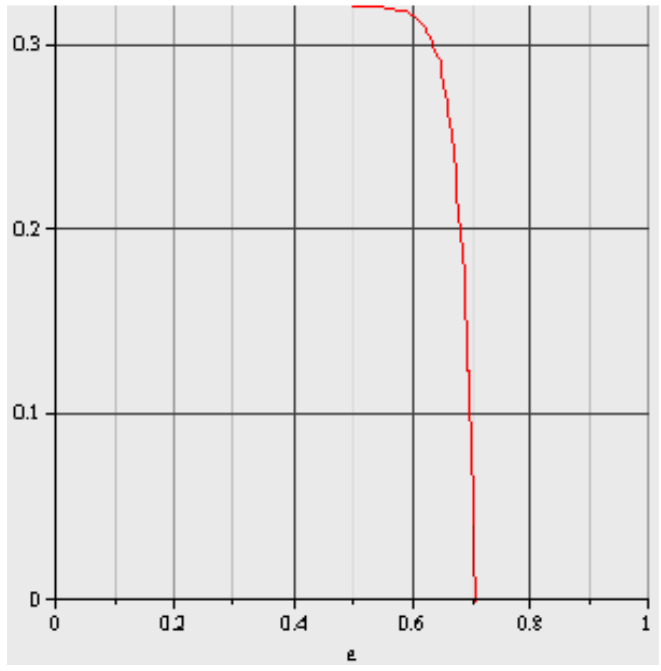

(b) $\quad N=40$

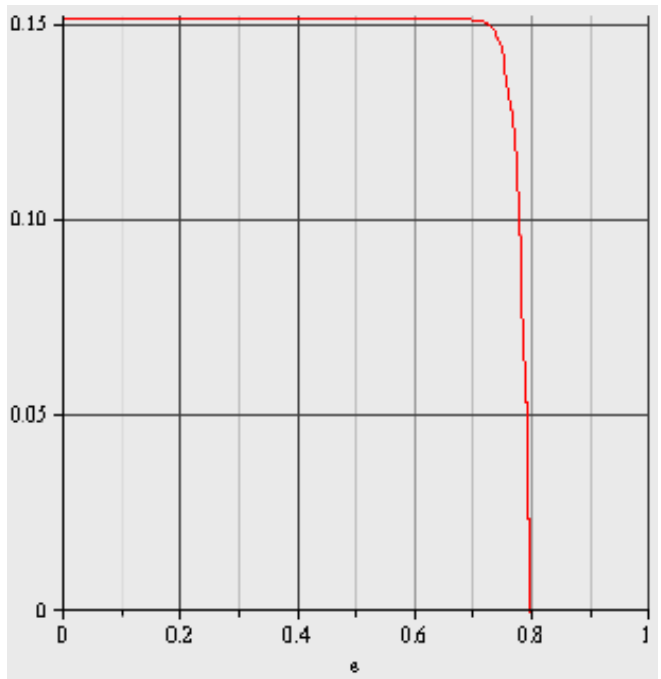

(d) $\quad N=60$

Figure 3. The optimal values of $\left(\delta_{j s}^{\sigma}\right)^{*}$ and $\left(W_{\sigma j s}^{(\hat{S})}\right)^{*}$ in 5 zones and different values of $N$.

If we divide the region into 10 zones, but with the same total number $N=30,40,50$ and 60 cells, then the optimal values $\left(\delta_{j s}^{\sigma}\right)^{*}$ and $\left(W_{\sigma j s}^{(\hat{S})}\right)^{*}$ are given in Table (1). These values obtained by the same method which used in the 5 zones case. Figure (4) presents the plotting $3 D$ of (22) and Figure (5) shows the optimal value of $\left(\delta_{j s}^{\sigma}\right)^{*}$ for 10 zones case. 


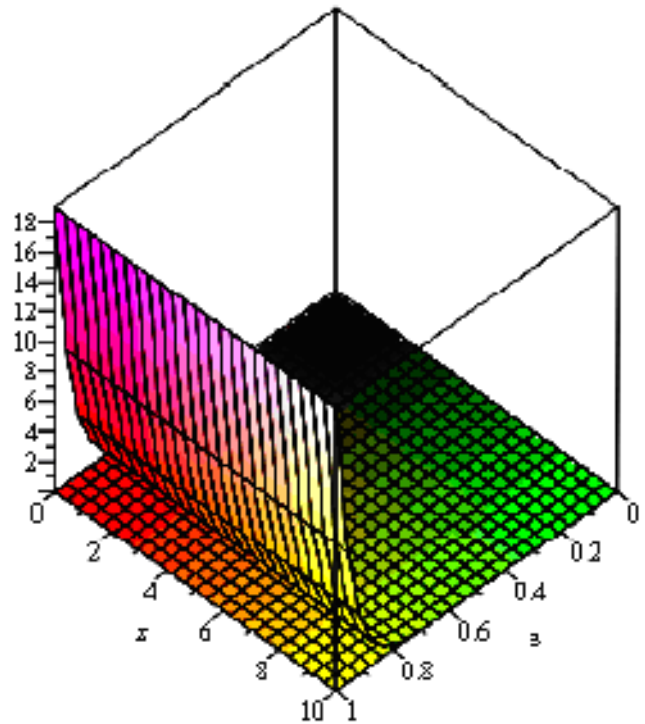

(a) $\quad N=30$

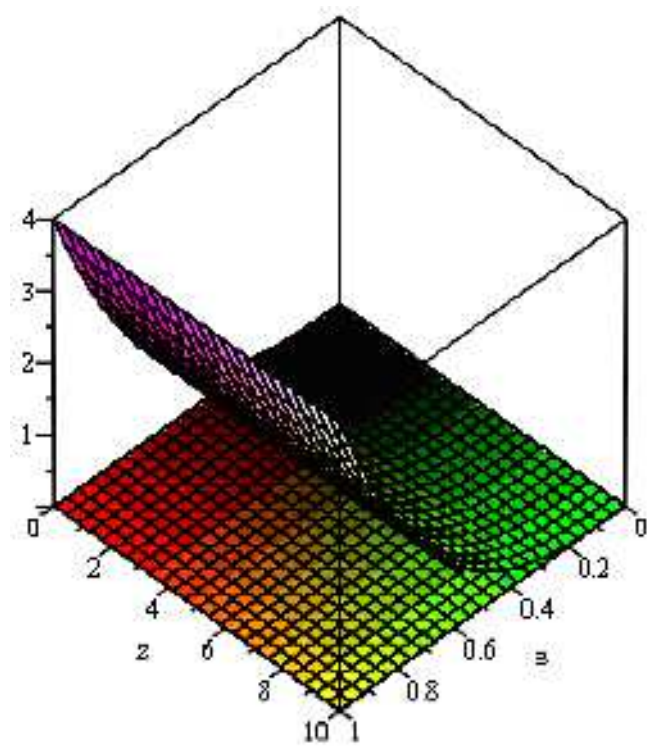

(c) $\quad N=50$

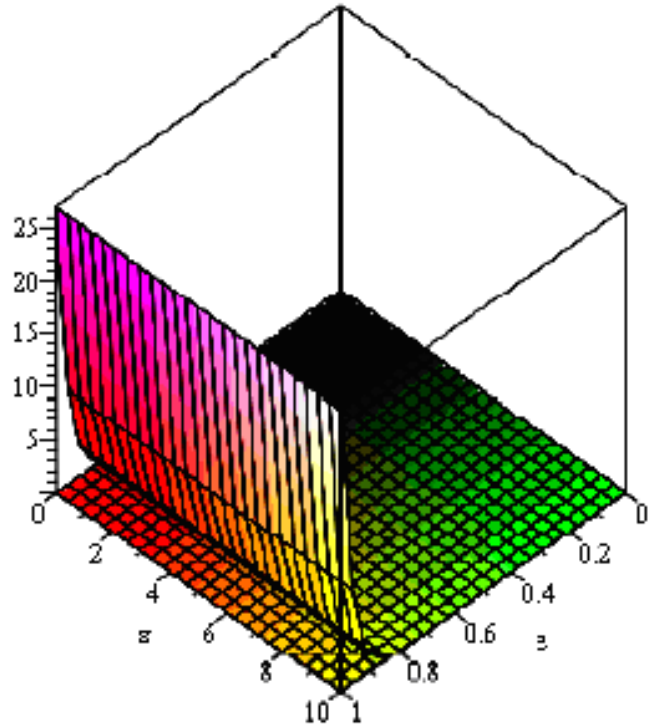

(b) $N=40$

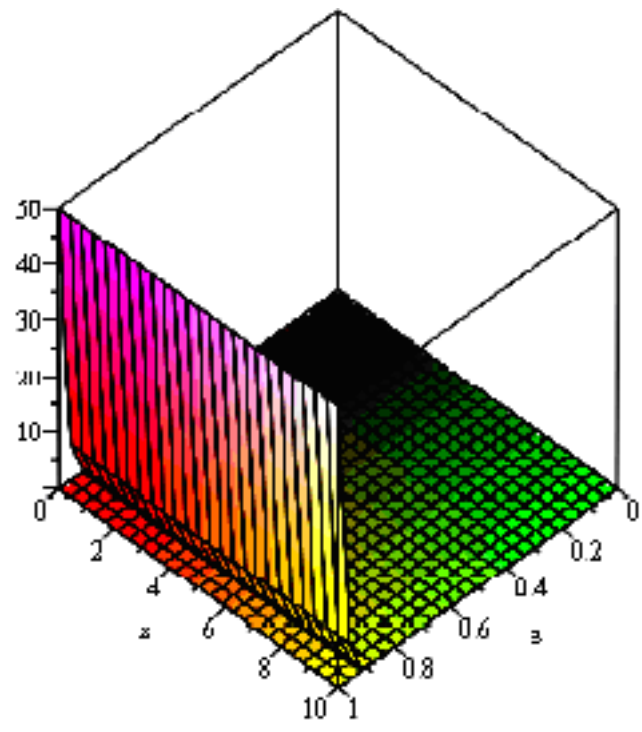

(d) $\quad N=60$

Figure 4. Plotting $3 D$ which presents the solution of (22) in 10 zones and different values of $N$. 


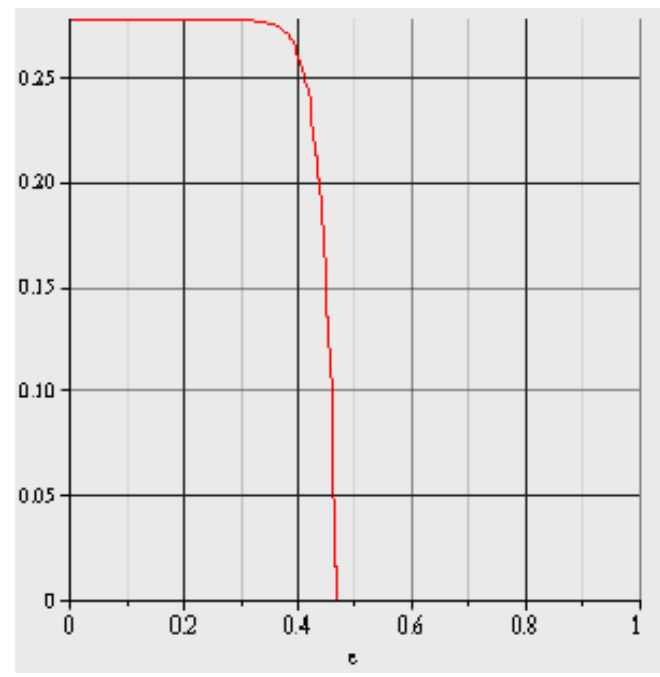

(a) $\quad N=30$

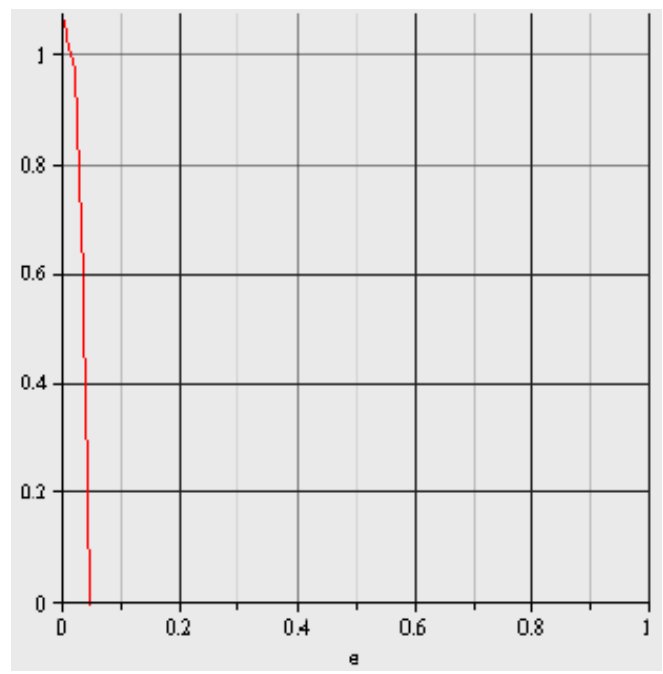

(c) $\quad N=50$

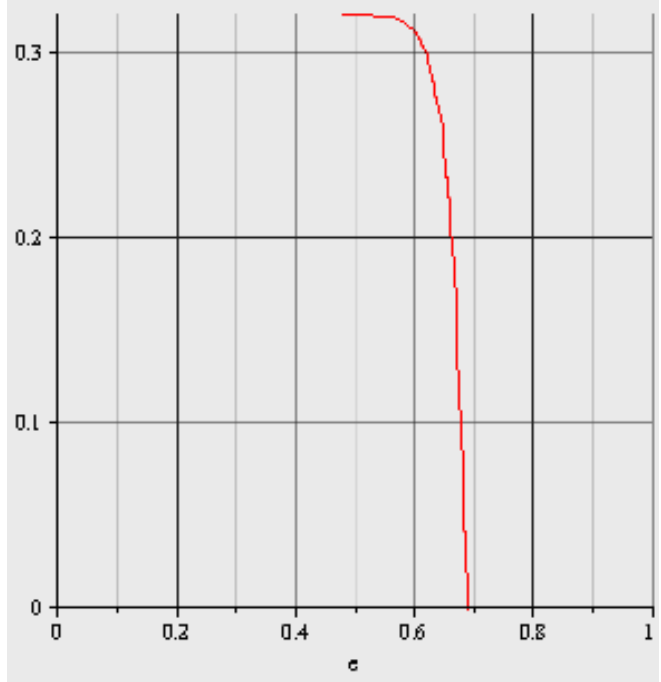

(b) $\quad N=40$

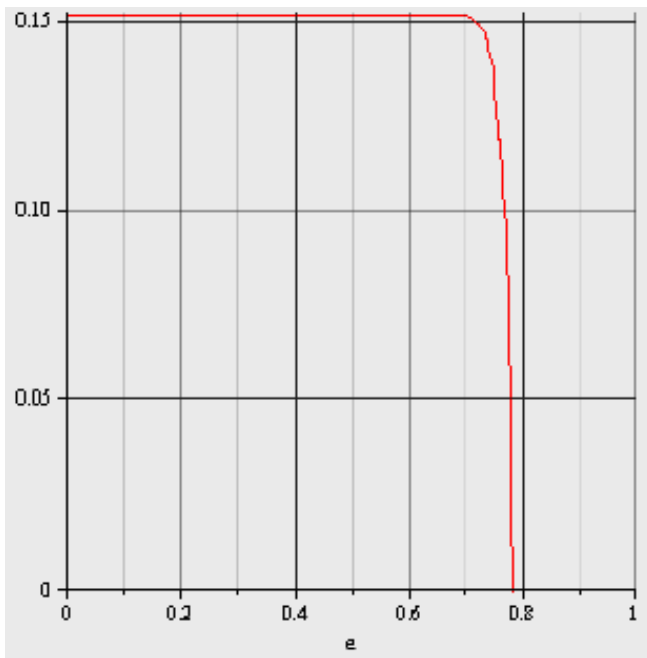

(d) $\quad N=60$

Figure 5. The optimal values of $\left(\delta_{j s}^{\sigma}\right)^{*}$ and $\left(W_{\sigma j s}^{(\hat{S})}\right)^{*}$ in 10 zones and different values of $N$.

\section{Concluding Remarks and the Future Work}

A novel probabilistic search model has been presented here to find the target with maximum probability and minimum search effort. The target move randomly on a known region, which divided into a finite number of zones. Each zone is divided also into a finite number of cells (not necessary identical). Also, each zone contains one sensor. The sensor aims to find the target with minimum effort and maximum probability. The effort is bounded by a known probability distribution.

We solve a difficult discrete stochastic optimization problem by using the discounted effort reward function. The solution of this problem can be obtained from the constructed algorithm which provided here. The effectiveness of 
this algorithm appeared in the numerical application. Moreover, this algorithm is more flexible where it is provided the optimal division of the region and then determined the optimal number of used sensors.

In the future work, we can extend our model to use multiple sensors in each zone. Also, we can solve this difficult discrete stochastic optimization problem under the fuzzy concept for the discounted effort reward.

\section{REFERENCES}

1. L. Stone, Theory of optimal search, 2nd Edition, Military Applications Section, Operations Research Soc of America, Arlington, VA. 1989.

2. S. Benkoski, M. Monticino, and J. Weisinger, A survey of the search theory literature, Naval Research Logistics, 38, 469-494, 1991.

3. G. M. Viswanathan, F. Bartumeus, Sergey V. Buldyrev, J. Catalan, U. L. Fulco, Shlomo Havlin, M. G. E. da Luz, M. L. Lyra, E. P. Raposo, and H. Eugene Stanley, Levy Flight Random Searches in Biological Phenomena, Physica A, 314, 208-213, 2000.

4. A. Beck, and D. Newman, Yet more on the linear search problem, Israel Journal of Mathematics, 8(4), 419-429, 1970.

5. A. Beck, and P. Warren, The return of the linear search problem, Israel Journal of Mathematics, 10(2), 169-183, 1972.

6. A. Mohamed, and M. El-Hadidy, Optimal multiplicative generalized linear search Plan for a discrete random walker, Journal of Optimization, Article ID 706176, 13 pages, 2013. http://dx.doi.org/10.1155/2013/706176.

7. A. B. EL- Rayes, A. A. Teamah, and H. M. Abou-Gabal, A linear search for a Brownian target motion, Acta Mathematica scienta, 23B, (3), 321-327, 2003

8. M. El-Hadidy, H. M. Abou-Gabal, Searching for the random walking microorganism cells, International Journal of Biomathematics, 12(6), 12, 2019, 1950064.

9. M. A. Kassem, A. A. Teamah, and M. El-Hadidy, Multiplicative linear search for a Brownian target motion, Applied Mathematical Modelling, 35, 4127-4139, 2011.

10. M. El-Hadidy, Searching for a d-dimensional Brownian target with multiple sensors, International Journal of Mathematics in Operational Research, 9(3), 279-301, 2016.

11. M. El-Hadidy, and A. Alzulaibani, Existence of a finite multiplicative search plan with random distances and velocities to find a ddimensional Brownian target, Journal of Taibah University for Science, 13(1), 1035-1043, 2019.

12. M. El-Hadidy, Generalised linear search plan for a D-dimensional random walk target, International Journal of Mathematics in Operational Research, 15(2), 211-241-, 2019.

13. M. El-Hadidy, Study on the three players linear rendezvous search problem, International Journal of Operational Research, 33(3), 297-314, 2018.

14. M. A. El-Hadidy, Existence of cooperative search technique to find a Brownian target, Journal of the Egyptian Mathematical Society, 28 (1), 1-12, 2020, Doi: 10.1186/s42787-019-0054-5.

15. M. El-Hadidy, and A. Alzulaibani, Cooperative search model for finding a brownian target on the real line, Journal of Taibah University for Science, 13(1), 177-183, 2019.

16. M. El-Hadidy, and H. Abou-Gabal, Coordinated Search for a Random Walk Target Motion, Fluctuation and Noise Letters, 17(1), 1850002 (11 pages), 2018.

17. D. J. Reyniers, Coordinated search for an object on the line, European Journal of Operational Research., 95(3), 663-670, 1996.

18. D. J. Reyniers, Coordinated two searchers for an object hidden on an interval, Journal of Operational Research Society, 46(11), 1386-1392, 1995.

19. A. Mohamed, H. Abou-Gabal, and M. El-Hadidy, Coordinated search for a randomly located target on the plane, European Journal of Pure and Applied Mathematics, 2(1), 97-111, 2009.

20. M. El-Hadidy, Detection of the Diffusion Nanoparticle in the Turbulent Flows Using the Random Walk Model, International Journal of Operational Research, In Press, 2020, https://www.inderscience.com/info/ingeneral/forthcoming.php?jcode=ijor.

21. M. El-Hadidy, and A. Alfreedi, On Optimal Coordinated Search Technique to Find a Randomly Located Target, Statistics, Optimization \& Information Computing, 7(4), 854-863, 2019.

22. M. El-Hadidy, H. Abou-Gabal, On the Linear Cooperative Search Technique for a Lost Target with Multiple Searchers, International Journal of Mathematics in Operational Research, In Press, 2021. https://www.inderscience. com/info/ingeneral/forthcoming.php?jcode=ijmor.

23. A. Mohamed, H. Fergany, and M. El-Hadidy, On the coordinated search problem on the plane, Istan Univ. J. Sch. Busin. Admin., 41(1), 80-102, 2012.

24. M. El-Hadidy, A. Teamah, and A. El-Bagoury, 3-Dimensional Coordinated search technique for a Randomly Located target, International Journal of Computing Science and Mathematics, 9(3), 258-272, 2018.

25. M. Kassem, and M. El-Hadidy, Optimal multiplicative Bayesian search for a lost target, Applied Mathematics and Computation, 247, 795-802, 2014.

26. F. Bourgault, T. Furukawa, and H. F. Durrant-Whyte, Optimal search for a lost target in a Bayesian world, S. Yaut et al. (EDS.):Field and Service Robotics, STAR 24, pp. 209-222, 2006.

27. M. El-Hadidy, and A. Alfreedi, Minimizing the expected search time of finding the hidden object by maximizing the discount effort reward search, Journal of Taibah University for Science, 14(1), 479-487, 2020.

28. M. El-Hadidy, Existence of Optimal N-Slinky-Turn-Spiral Search Paths for Finding N-Dimensional Brownian Target,International Journal of Mathematics in Operational Research, In Press, 2020, https://www.inderscience.com/info/ingeneral/forthcoming.php?jcode=ijmor.

29. M. El-Hadidy, Fuzzy Optimal Search Plan for N-Dimensional Randomly Moving Target, International Journal of Computational Methods, 13 (6), 2019, 1650038 (38 pages). 
30. M. El-Hadidy, and M. A. Alzulaibani, A mathematical model for preventing HIV virus from proliferating inside CD4 T brownian cell using Gaussian jump nanorobot, International Journal of Biomathematics, 12(07), 24 pages, 2019, 1950076.

31. M. El-Hadidy, and A. Alzulaibani, Study on the finiteness of the first meeting time between N-dimensional Gaussian jump and Brownian diffusion particles in the fluid, International Journal of Modern Physics B. 33(28), 22 pages, 2019, 1950334.

32. A. Mohamed, and M. El-Hadidy, On probabilistic modeling and feasibility of collision between a randomly moving meteor and satellite, Afrika Matematika, 32(1-2), 1-15, 2021. Doi: 10.1007/s13370-018-0591-3.

33. M. El-Hadidy, Existence of finite parbolic spiral search plan for a Brownian target, International Journal of Operational Research, 31(3), 368-383, 2021.

34. M. El-Hadidy, Studying the finiteness of the first meeting time between Lévy flight jump and Brownian particles in the fluid reactive anomalous transport, Modern Physics Letters B, 33(22), 1950256, 8 pages, 2019.

35. M. El-Hadidy, Quality control for a detected an appropriate queue from K-independent M/M/C/N queueing models, , Quality and Reliability Engineering International, 37(1), 165-175, 2021.

36. A. Mohamed, H. Abou Gabal, and M. El-Hadidy, Random search in a bounded area, International Journal of Mathematics in Operational Research, 10(2), 137-149, 2017.

37. H. Abou-Gabal, M. Hamdy, and M. A. El-Hadidy, Optimal searching for a randomly located target in a bounded known region, International Journal of Computing Science and Mathematics, Vol. 6, No. 4, 392-402, 2015.

38. M. El-Hadidy, On Maximum Discounted Effort Reward Search Problem, Asia-Pacific Journal of Operational Research, 33(3), 30 pages, 2016, 1650019 .

39. M. El-Hadidy, The Searching Algorithm for Detecting a Markovian Target Based on Maximizing the Discounted Effort Reward Search, Journal of the Egyptian Mathematical Society, 28(37), 1-18, 2020. DOI:10.1186/s42787-020-00097-1.

40. A. A. Teamah, M. A. Kassem, and M. A. El-Hadidy, Mstates search problem for a lost target with multiple sensors, International Journal of Mathematics in Operational Research, 10(1), 104-135, 2017.

41. S. Hong, S. Cho, and M. Park, A pseudo-polynomial heuristic for pathconstrained discrete-time Markovian-target search, European Journal of Operational Research, 193, 351-364, 2009.

42. M. El-Hadidy, Spiral With Line Segment Directory for a Helix Search Path to Find a Randomly Located Target in the Space, International Journal of Operational Research, 40(2), 185-199, 2021.

43. M. El-Hadidy, and M. Fakharany, Optimal 3-Dimensional Search Model to Find the Underwater Randomly Hidden Target, International, Journal of Mathematics in Operational Research, 18(2), 210-235, 2021.

44. M. El-Hadidy, Developing a detection model for a COVID-19 infected person based on a probabilistic dynamical system, Mathematical Methods in the Applied Sciences, In Press, 2021. Doi: 10.1002/mma.7443.

45. M. El-Hadidy, A. Alfreedi, Detection of an appropriate pharmaceutical company to get a suitable vaccine against COVID-19 with minimum cost under the quality control process, Quality and Reliability Engineering International, In Press. 2021, Doi: 10.1002/qre.2881. 